Canad. Math. Bull. Vol. 22 (1), 1979

\title{
ON A CLASS OF POLYNOMIALS ASSOCIATED WITH THE STARS OF A GRAPH AND ITS APPLICATION TO NODE-DISJOINT DECOMPOSITIONS OF COMPLETE GRAPHS AND COMPLETE BIPARTITE GRAPHS INTO STARS
}

\author{
BY \\ E. J. FARRELL
}

\begin{abstract}
A star is a connected graph in which every node but possibly one has valency 1 . Let $G$ be a graph and $C$ a spanning subgraph of $G$ in which every component is a star. With each component $\alpha$ of $C$ let us associate a weight $w_{\alpha}$. Let $\prod_{\alpha} w_{\alpha}$ be the weight associated with the entire subgraph $C$. the star polynomial of $G$ is $\sum \prod_{\alpha} w_{\alpha}$, where the summation is taken over all spanning subgraphs of $G$ consisting of stars. In this paper an algorithm for finding star polynomials of graphs is given. The star polynomials of various classes of graphs are then found, and some results about node-disjoint decomposition of complete graphs and complete bipartite graphs are deduced.
\end{abstract}

1. Introduction. A star is a connected graph in which every node, except possibly one, has valency 1 . By an $m$-star $(m \geq 0)$ we shall mean a star which contains $m$ edges. When $m>1$ we call the node of valency greater than 1 -the centre of the star.

Let $G$ be a graph. By a cover of $G$ we shall mean a spanning subgraph of $G$. A star cover of $G$ is a cover whose components are all stars. Since the only covers which will be considered here are star covers, we shall use the word "cover" to mean "star cover" unless otherwise specified. Let us associate a weight $w_{\alpha}$ with the component $\alpha$ of a cover $C$ of $G$, and let $\prod_{\alpha} w_{\alpha}$ be the weight associated with the entire cover. Then the star polynomial of $G$ is $\sum \prod_{\alpha} w_{\alpha}$, where the summation is taken over all covers of $G$. The star polynomial is therefore a special case of the F-polynomial defined in Farrell [3].

In this paper, we will assign the same weight to all components with the same number of nodes. Therefore an $m$-star will be assigned the weight $w_{m+1}$. The star polynomial of $G$ is therefore a polynomial in the indeterminates $w_{1}, w_{2}, w_{3}, \ldots$ etc. Let $\mathbf{w}=\left(w_{1}, w_{2}, w_{3}, \ldots\right)$ be a vector of indeterminates. The star polynomial of $G$ will be denoted by $E(G ; \mathbf{w})$. Its terms will be of the form $A w_{1}^{a} w_{2}^{b} w_{3}^{c} \cdots$ where $A$ is the number of covers of $G$ consisting of a 0 -stars

Received by the editors June 15, 1977 and, in revised forms, March 28, 1978 and May 31, 1978.

Classification AMS(MOS) (1970) 05C10, 05C99

Keywords and Phrases. Graph; star; star polynomial; decomposition of a graph; generating function; node disjoint subgraphs. 
(isolated nodes), $b$ 1-stars (independent edges), $c$ 2-stars etc. If we put $w_{i}=w$ for all $i$, then the resulting polynomial in $w$ will be called the simple star polynomial of $G$, and wil be denoted by $E(G ; w)$. In order to avoid ambiguity between this polynomial and the polynomial in $w_{i}$, we will call $E(G ; \mathbf{w})$ the general star polynomial of $G$, whenever both polynomials are being found for the same graph.

We shall denote generating functions of $E(G ; \mathbf{w})$ by $E(G ; \mathbf{w}, t)$ and $E(G ; w, u, v)$, where the extra variables $t, u$ and $v$ serve to collect covers with common parameters; for example, number of nodes. The lower limits of all summations will be zero unless otherwise specified. The upper limits will be infinity, or the maximum value of the variable which will make sense in the context of the summand.

We will give algorithms for finding star polynomials of graphs. Basic results of these polynomials will also be given. We will then derive the polynomials for special kinds of trees, circuits, complete graphs and complete bipartite graphs. As corollaries of our main results we will give some results concerning node-disjoint decompositions of complete graphs and complete bipartite graphs into stars. We refer the reader to Harary [4] for the basic definitions in Graph Theory.

2. The fundamental algorithm. Let $G$ be a graph and $x y$ a given edge of $G$. Let $v_{x}$ and $v_{y}$ denote the valencies of $x$ and $y$ respectively, where $v_{x}>1$ and $v_{y}>1$. We can divide the covers of $G$ into four classes; (1) those which do not contain $x y$, (2) those in which $x y$ is a component, (3) those in which $x$ is the centre of an $m$-star $(m>1)$ and (4) those in which $y$ is the centre of an $m$-star $(m>1)$. The covers in class (1) are covers of the graph $G^{\prime}$ obtained from $G$ by deleting the edge $x y$. The covers in class (2) are covers of the graph $G-\{x, y\}$ obtained from $G$ by removing nodes $x$ and $y$ (and their adjacent edges), with the edge $x y$ added to each cover. The covers in class (3) are covers of the graph $G_{x}$ obtained from $G$ by distinguishing $x$ in some way and requiring it to be the centre of an $m$-star $(m>1)$ in every cover of $G_{x}$. The covers in class (4) are covers of the graph $G_{y}$ obtained from $G$ by distinguishing $y$ in some way and requiring it to be the centre of an $m$-star $(m>1)$ in every cover of $G_{y}$. Our discussion leads to the following theorem.

THEOREM 1. Let $G$ be a graph and $x y$ a given edge of $G$ such that the valencies of both $x$ and $y$ are greater than unity. Then

$$
E(G ; \mathbf{w})=E\left(G^{\prime} ; \mathbf{w}\right)+w_{2} E(G-\{x, y\} ; \mathbf{w})+E\left(G_{x} ; \mathbf{w}\right)+E\left(G_{y} ; \mathbf{w}\right) .
$$

One method of finding $E\left(G_{x} ; \mathbf{w}\right)$ is to remove $x$ and its adjacent nodes from $G$ to form the graph $G_{x}^{\prime}$, and then to multiply $E\left(G_{x}^{\prime} ; \mathbf{w}\right)$ by the contribution $\sum_{k=3}^{v_{x}} w_{k} w_{1}^{v_{x}-k}$ of the sub-covers in which node $x$ is the centre of a star with 
more than one edge, i.e. $x$-rooted coverings. We therefore give an explicit algorithm in the following corollary.

Corollary 1.1.

$$
\begin{aligned}
E(G ; \mathbf{w})=E\left(G^{\prime} ; \mathbf{w}\right)+w_{2} E(G-\{x, y\} ; \mathbf{w}) & \\
& +\left(\sum_{k=3}^{v_{x}} w_{k} w_{1^{v_{x}}-k}^{v^{2}}\right) E\left(G_{x}^{\prime} ; \mathbf{w}\right)+\left(\sum_{k=3}^{v_{y}} w_{k} w_{1^{y}}^{v_{y}-k}\right) E\left(G_{y}^{\prime} ; \mathbf{w}\right) .
\end{aligned}
$$

The fundamental algorithm for star polynomials consists of repeated applications of Theorem 1 until we obtain graphs $H_{i}$ for which $E\left(H_{i} ;\right.$ w) are known. In the case where node $x$ (or node $y$ ) has valency 1 , the following result is immediate from Corollary 1.1.

Corollary 1.2. If $x$ is a node of valency 1 in a graph $G$, and $y$ is the node adjacent to $x$, then

$$
E(G ; \mathbf{w})=w_{1} E(G-\{x\} ; \mathbf{w})+w_{2} E(G-\{x, y\} ; \mathbf{w})+\left(\sum_{k=3}^{v_{y}} w_{k}\right) w_{1} E\left(G_{y}^{\prime} ; \mathbf{w}\right) .
$$

3. Some basic results about star polynomials. Some of the results given here will be useful in applications of the fundamental algorithm. We define the star polynomial of the null graph to be the integer 1 . It is clear that for the isolated node $G, E(G ; \mathbf{w})=w_{1}$ and if $G$ consists of two nodes joined by an edge, then $E(G ; \mathbf{w})=w_{1}^{2}+w_{2}$.

$r$ If $G$ consisted of two components $H_{1}$ and $H_{2}$, then every cover of $G$ would be the union of a cover of $H_{1}$ and a cover of $H_{2}$ and vice versa. Thus we have the following result, called the Component Theorem.

THEOREM 2 (The Component Theorem). If $G$ consists of components $H_{1}, H_{2}, \ldots, H_{k}$, then

$$
E(G ; \mathbf{w})=\prod_{i=1}^{k} E\left(H_{i} ; \mathbf{w}\right) .
$$

The following theorem gives some of the basic properties of star polynomials.

THEOREM 3. Let $G$ be a graph with $p$ nodes and $q$ edges. Then

(i) Each term of $E(G ; \mathbf{w})$ is of the form $A w_{1}^{n_{1}} w_{2}^{n_{2}} \cdots w_{p}^{n_{p}}$, where $A$ is a non-negative integer and $\sum_{i=1}^{p} i n_{i}=p$.

(ii) The coefficient of $w_{1}^{p}$ in $E(G: w)$ is 1 .

(iii) The coefficient of $w_{1}^{p-2} w_{2}$ in $E(G ; w)$ is $q$.

(iv) $E(G ; \mathbf{w})$ contains no constant term when $G$ is nonnull.

(v) If the lowest power of $w_{1}$ in $E(G ; w)$ is $w_{1}^{r}$ then the highest valency of a node in $G$ is $p-r$. 
4. Star polynomials of trees. The star polynomial of a given tree can be found by using the reduction process or Corollary 1.2. However finding the star polynomial of a general tree would be a formidable task, since there are several trees with any given number of nodes. We can however obtain results for special kinds of trees.

By a chain we will mean a tree with nodes of valencies 1 and 2 only. The chain with $p$ nodes will be denoted by $P_{p}$. Therefore $P_{0}$ is the null graph, $P_{1}$ is an isolated node and $P_{2}$ is a 2 -star. We can apply Corollary 1.2 to $P_{p}$ by taking $x y$ to be a terminal edge of $P_{n}$. In this case $G-\{x\}$ will be $P_{p-1}, G-\{x, y\}$ will be $P_{p-2}$ and $G_{y}^{\prime}$ will be $P_{p-3}$. Hence we have the following lemma, in which $P(p)$ is temporarily written for $E\left(P_{p} ; \mathbf{w}\right)$.

LEMMA 1.

$$
P(p)=w_{1} P(p-1)+w_{2} P(p-2)+w_{3} P(p-3) \quad(p>2),
$$

where

$$
P(0)=1, \quad P(1)=w_{1} \quad \text { and } \quad P(2)=w_{1}^{2}+w_{2} .
$$

We can use the recurrence relation given in Lemma 1 in order to obtain an ordinary generating function $E\left(P_{p} ; \mathbf{w}, t\right)$ for $E\left(P_{p} ; \mathbf{w}\right)$. From the coefficient of $t^{p}$ an explicit formula for $P(p)$ can be obtained. Thus we have

\section{THEOREM 4.}

$$
E\left(P_{p} ; \mathbf{w}\right)=\sum \frac{(a+b+c) !}{a ! b ! c !} w_{1}^{a} w_{2}^{b} w_{3}^{c}
$$

where the summation is taken over all solutions of $a+2 b+3 c=p$, and its ordinary generating function is

$$
E\left(P_{p} ; \mathbf{w}, t\right)=\left(1-w_{1} t-w_{2} t^{2}-w_{3} t^{3}\right)^{-1} .
$$

Let us denote the $m$-star by $S_{m}$. We can apply Corollary 1.2 to $S_{m}$ by taking $y$ as the centre of the star. Then $G-\{x\}$ will be $S_{m-1}, G-\{x, y\}$ will be $m-1$ isolated nodes and $G_{y}^{\prime}$ will be the null graph. In this case $v_{y}=m$. Therefore we obtain the following recurrence, in which $S(m)$ is temporarily written for $E\left(S_{m} ; \mathbf{w}\right)$.

$$
S(m)=w_{1} S(m-1)+w_{1}^{m-1} w_{2}+w_{m+1}(m>0) \text { and } S(0)=w_{1} .
$$

We can obtain an explicit formula for $E\left(S_{m} ; \mathbf{w}\right)$ by the following considerations. Any cover of $S_{m}$ must consist of a $k$-star and $m-k$ isolated nodes. Since any choice of $k$ edges yields a $k$-star, the number of covers consisting of a $k$-star and $m-k$ isolated nodes is $\left(\begin{array}{c}m \\ k\end{array}\right)$. Hence we have the following theorem. 
THEOREM 5.

$$
E\left(S_{m} ; \mathbf{w}\right)=\sum_{k=0}^{m}\left(\begin{array}{l}
m \\
k
\end{array}\right) w_{1}^{m-k} w_{k+1} .
$$

We can use the above recurrence for $S(m)$ in order to obtain its ordinary generating function. This is given in the following result.

THEOREM 6.

$$
E\left(S_{m} ; w, t\right)=\frac{w_{1}-w_{1}^{2} t-w_{2} t+\left(1-w_{1} t\right) w(t)}{\left(1-w_{1} t\right)^{2}}
$$

where

$$
w(t)=\sum_{k=1} w_{k+1} t^{k}
$$

5. Star polynomials of circuits. Let us denote the circuit with $p$ nodes by $C_{p}$. We can apply Corollary 1.1 to $C_{p}$ by deleting any edge $x y$. In this case $G^{\prime}$ will be the chain $P_{p}, G-\{x, y\}$ will be the chain $P_{p-1}, G_{x}^{\prime}$ will be $P_{p-3}$ and $G_{y}^{\prime}$ will be $P_{n-3}$. Both $x$ and $y$ will have valency 2 , so $v_{x}=v_{y}=2$. We therefore get the following lemma, in which $C(p)$ and $P(p)$ are written for $E\left(C_{p} ; \mathbf{w}\right)$ and $E\left(P_{p} ; \mathbf{w}\right)$ respectively.

Lemma 3.

$$
C(p)=P(p)+w_{2} P(p-2)+2 w_{3} P(p-3) \quad(p>2),
$$

where

$$
P(0)=1, \quad P(1)=w_{1} \quad \text { and } \quad P(2)=w_{1}^{2}+w_{2} .
$$

We can use the generating function for $P(p)$ given in Theorem 4 in order to obtain a generating function for $C(p)$. An explicit formula for $C_{p}$ could be obtained either directly from Lemma 3 by using the formula for $P(p)$ given in Theorem 4 , or from the generating function for $C(p)$. A generating function $E\left(C_{p} ; w, t\right)$ for $C(p)$ and an explicit formula for $C(p)$, is given in the following theorem. (N.B. We take $C_{2}$ to be the multigraph consisting of two nodes joined by two edges.)

TheOREM 7. The generating function $E\left(C_{p} ; \mathbf{w}, t\right)$ for $E\left(C_{p} ; \mathbf{w}\right)$ is

$$
\begin{gathered}
E\left(C_{p} ; w, t\right)=\left(1+w_{2} t^{2}+2 w_{3} t^{3}\right)\left(1-w_{1} t-w_{2} t^{2}-w_{3} t^{3}\right)^{-1} \quad(p>2), \\
C(0)=1, \quad C(1)=w_{1} \quad \text { and } \quad C(2)=w_{1}^{2}+2 w_{2} .
\end{gathered}
$$


Also

$$
\begin{aligned}
E\left(C_{p} ; w\right)=\sum \frac{(a+b+c) !}{a ! b ! c !} & w_{1}^{a} w_{2}^{b} w_{3}^{c} \\
& +\sum \frac{(a+b+c) !}{a ! b ! c !} w_{1}^{a} w_{2}^{b+1} w_{3}^{c}+2 \sum \frac{(a+b+c) !}{a ! b ! c !}+w_{1}^{a} w_{2}^{b} w_{3}^{c+1},
\end{aligned}
$$

where the summations are taken over all solutions of $a+2 b+3 c=p$, $a+2 b+3 c=p-2$ and $a+2 b+3 c=p-3$ respectively.

6. Star polynomials of complete graphs. Let us denote the complete graph with $p$ nodes by $K_{p}$. In any cover of $K_{p}$ either (i) $x$ is an isolated node, (ii) $x$ is in a 1 -star or (iii) $x$ is in an $i$-star for $i>1$. In case (i) the contribution of the isolated node $x$ to $K(p) \equiv E\left(K_{p} ; w\right)$ is $w_{1}$, while the remaining elements of the cover will be a cover of the graph $K_{p-1}$. Therefore the contribution of this class of covers to $K(p)$ is $w_{1} K(p-1)$. If $x$ is in a 1-star, it can be in the 1-star in only one way, since the nodes of a 1-star are identical. However there are $p-1$ 1 -stars that will contain $x$. The remaining elements of the cover will be a cover of $K_{p-2}$. Hence the contribution of this class of covers to $K(p)$ is $(p-1) \times$ $w_{1} K(p-2)$. In case (iii) the weight of the $i$-star will be $w_{i+1}$. There are $i+1$ ways in which $x$ could be in an $i$-star. It can either be the centre or one of the $i$ nodes of valency 1 . Also, there will be $\left(\frac{p-1}{i}\right)$ ways of choosing $i$ nodes adjacent to $x$. The remaining elements of the cover will be a cover of $K_{p-i-1}$. Therefore the contribution of this class of covers to $K(p)$ is $\left({ }_{i}^{p-1}\right) i w_{i+1} K(p-i-1)$. In summary, we get the following result, in which $K(p)$ is written for $E\left(K_{p} ; \mathbf{w}\right)$.

LEMMA 4.

$$
\begin{gathered}
K(p)=w_{i} K(p-1)+(p-1) w_{2} K(p-2)+\sum_{i=3}^{p}\left(\begin{array}{c}
p-1 \\
i-1
\end{array}\right) i w_{i} K(p-i) \quad(p>1), \\
K(0)=1, \quad K(1)=w_{1} \text { and } K(2)=w_{1}^{2}+w_{2} .
\end{gathered}
$$

We can use this recurrence relation in order to obtain an exponential generating function $E\left(K_{p} ; \mathbf{w}, t\right)$ for $E\left(K_{p} ; \mathbf{w}\right)$. By extracting the coefficient of $t^{p}$ and multiplying by $p$ !, an explicit formula for $E\left(K_{p} ; \mathbf{w}\right)$ is obtained. These results are given in the following theorem.

TheOREM 8.

$$
E\left(K_{P} ; \mathbf{w}, t\right)=\exp \left[w_{1} t+w_{2} \frac{t^{2}}{2}+w(t)\right]
$$

where

$$
w(t)=\sum_{i=3} \frac{w_{i} t^{i}}{(i-1) !}
$$


Therefore

$$
E\left(K_{p} ; \mathbf{w}\right)=p ! \sum\left[\frac{1}{j_{2} !}\left(\frac{w_{2}}{2}\right)^{s_{2}} \prod_{i \neq 2} \frac{1}{j_{1} !}\left(\frac{w_{i}}{(i-1) !}\right)^{j_{i}}\right],
$$

where the summation is taken over all solutions of $\sum_{i} i j_{i}=p$.

We can obtain analogous results for the simple star polynomial of $K_{p}$ by putting $\mathbf{w}=(w, w, w, \ldots)$ in Theorem 9 and Lemma 4 . By making this substitution in $E\left(K_{p} ; \mathbf{w}, t\right)$ we get

$$
\begin{aligned}
E\left(K_{p} ; w, t\right) & =\exp \left[w t+w \frac{t^{2}}{2}+\sum_{i=3} \frac{w t^{i}}{(i-1) !}\right] \\
& =\exp \left\{w t\left[\sum_{i}\left(\frac{t^{i}}{i !}\right)-\frac{t}{2}\right]\right\} .
\end{aligned}
$$

Thus we have

COROLlaRY 8.1. The generating function for the simple star polynomial of $K_{p}$ is

$$
E\left(K_{p} ; w, t\right)=\exp \left[w t\left(\exp t-\frac{t}{2}\right)\right] .
$$

We can obtain a formula for $E\left(K_{p} ; w\right)$ from Theorem 9 . However, a more explicit formula can be obtained from the above generating function. We have

$$
\begin{aligned}
E\left(K_{p} ; w, t\right) & =\exp (w t \exp t) \exp \left(-w \frac{t^{2}}{2}\right) \\
& =\sum_{r} \frac{w^{r} t^{r} e^{r t}}{r !} \cdot \sum_{s}(-1)^{s} \frac{w^{s} t^{2 s}}{2^{s} s !} \\
& =\sum_{r}\left[\frac{w^{r} t^{r}}{r !} \sum_{i} \frac{r^{i} t^{i}}{i !}\right] \cdot \sum_{s}(-1)^{s} \frac{w^{s} t}{2^{s} s !} \\
& =\sum_{r} \sum_{i} \sum_{s}(-1)^{s} \frac{w^{r+s} r^{i} t^{r+i+2 s}}{r ! i ! s ! 2^{s}} .
\end{aligned}
$$

By equating coefficients of $t^{p}$ and multiplying by $p$ !, we get

$$
E\left(K_{p} ; w\right)=p ! \sum_{r, s} \frac{(-1)^{s} r^{p-r-2 s} w^{r+s}}{r !(p-r-2 s) ! s ! 2^{s}} .
$$

By putting $r+s=k$, we obtain

THEOREM 9.

$$
E\left(K_{p} ; w\right)=p ! \sum_{k}^{p} \frac{w^{k}}{2^{k} k !} \sum_{r}^{k}(-1)^{k-r}\left(\begin{array}{l}
k \\
r
\end{array}\right) \frac{2^{r} r^{p+r-2 k}}{(p+r-2 k) !} .
$$

An immediate corollary is the following. 
COROLlaRy 9.1. The number of spanning forests on $p$ labelled nodes that consist of $k$ stars is

$$
\frac{p !}{2^{k} k !} \sum_{r}^{k}\left(\begin{array}{l}
k \\
r
\end{array}\right)(-1)^{k-r} \frac{2^{r} r^{p+r-2 k}}{(p+r-2 k) !} .
$$

7. Node-disjoint decomposition of complete graphs into stars. Recall from Theorem 8 that

$$
E\left(K_{p} ; \mathbf{w}\right)=p ! \sum \frac{1}{j_{2} !}\left(\frac{w_{2}}{2}\right)^{j_{2}} \prod_{i \neq 2} \frac{1}{j_{i} !}\left(\frac{w_{i}}{(i-1) !}\right)^{j_{i}}
$$

where the summation is taken over all solutions of $\sum_{i} i j_{i}=p$.

An $m$-star decomposition of a graph $G$ is a cover of $G$ in which every component is an $m$-star. We can therefore obtain the number of $m$-star $(m>1)$ decompositions of $K_{p}$, by putting $j_{m+1}=p /(m+1)$ and $j_{i}=0$ for $i \neq m+1$. Thus we obtain the following corollary.

Corollary 8.2. If $K_{p}$ has an $m$-star decomposition, then $m+1$ divides $p$, and then the number of such decompositions is

$$
\begin{aligned}
& \frac{p !}{r !(m !)^{r}}, \quad \text { if } m \neq 1 \text { and } p=(m+1) r \\
& \frac{p !}{(r !) 2\left(2^{r}\right)} \text { if } m=1 \text { and } p=2 r .
\end{aligned}
$$

The number of decompositions of $K_{p}$ into stars of a given minimum size is given in the following result.

COROLlaRy 8.3. The number of decompositions of $K_{p}$ into stars which are all bigger than an $m$-star $(m \neq 1)$ is

$$
p ! \sum \prod_{i>m+1} \frac{1}{j_{i} !}\left(\frac{1}{(i-1) !}\right)^{j_{i}}
$$

where the summation is taken over all solutions of $\sum_{i} i j_{i}=p$ in which $i$ is greater than $m+1$.

The following result can be easily obtained from Theorem 8 .

COROLlaRY 8.4. The number of decompositions of $K_{p}$ into $m_{1}$-stars, $m_{2}$ stars, ..., $m_{r}$-stars $\left(m_{i} \neq 1\right.$ for any $\left.i\right)$ is

$$
p ! \sum \frac{1}{\prod_{i=1}^{r} j_{m_{i}}\left(m_{i} !\right)^{j_{m_{i}}}},
$$

where the summation is taken over all solutions of

$$
\sum_{i} j_{m_{i}}\left(m_{i}+1\right)=p
$$


COROllaRy 8.5. The number of decompositions of $K_{p}$ into $n_{1} m_{1}$-stars, $n_{2} m_{2}$ stars $\cdots$ and $n_{r} m_{r}$-stars, with $m_{i} \neq 1$ for all $i$, and $\sum_{i} n_{i}\left(m_{i}+1\right)=p$, is

$$
\frac{p !}{\prod_{i=1}^{r} n_{i} !\left(m_{i} !\right)^{n_{i}}} .
$$

The edge-decomposition of $K_{p}$ into stars is discussed in Cain [2] and Ae et al. [1].

8. Star polynomials of complete bipartite graphs. Let us denote the complete bipartite graph with bipartition $m$ and $n$ by $K_{m, n}$. We will use a combinatorial technique in order to find $E\left(K_{m, n} ; \mathbf{w}\right)$-the star polynomial of $K_{m, n}$.

Let us divide the covers of $K_{m, n}$ into two classes: (i) those in which a particular node $x$ is the centre of a star and (ii) those in which $x$ is not the centre of a star. We will call the non-adjacent nodes in the set with cardinality $m$, red nodes, and those in the set with cardinality $n$, black nodes. Without loss in generality, we will assume that node $x$ is a red node.

Consider a cover in class 1 . Suppose that $x$ is the centre of a $j$-star. Then node $x$ will be joined to $j$ black nodes. There will be $\left(\begin{array}{l}n \\ j\end{array}\right)$ ways of choosing these nodes. The weight of a $j$-star is $w_{j+1}$. The other elements of the cover will be a cover of $K_{m-1, n-j}$. Hence the contribution of this cover to $E\left(K_{m, n} ; w\right)$ will be $\left(\begin{array}{l}n \\ j\end{array}\right) w_{j+1} E\left(K_{m-1, n-j} ; \mathbf{w}\right)$. Thus the total contribution of all the covers in class 1 will be

$$
C_{1}=\sum_{j}\left(\begin{array}{l}
n \\
j
\end{array}\right) w_{j+1} E\left(K_{m-1, n-j} ; \mathbf{w}\right) .
$$

By writing $r=n-j$ and $E(m, n) \equiv E\left(K_{m, n} ; \mathbf{w}\right)$, we get

$$
C_{1}=\sum_{r}\left(\begin{array}{l}
n \\
r
\end{array}\right) w_{n-r+1} E(m-1, r) \text {. }
$$

Let us consider the covers in class 2 . Since node $x$ cannot be the centre of a star, we will assume that $x$ is in a $j$-star, $(j>1)$ whose centre is a black node. Let us choose a black node. This could be done in $n$ ways. This node could be the centre of a $j$-star containing $x$ in $\left(\begin{array}{c}m-1 \\ j-1\end{array}\right)$ ways, since we must choose $j-1$ other red nodes to join to the black node. Hence the contribution of the covers in which $x$ is part of a $j$-star $(j>1)$ is

$$
n\left(\begin{array}{c}
m-1 \\
j-1
\end{array}\right) w_{j+1} E(m-j, n-1) .
$$

Thus the contribution to $E(m, n)$ of all the covers in class 2 is

$$
C_{2}=n \sum_{j}\left(\frac{m-1}{j-1}\right) w_{j+1} E(m-j, n-1)
$$


By putting $s=m-j$, we get

$$
C_{2}=n \sum_{s}^{m-2}\left(\begin{array}{c}
m-1 \\
s
\end{array}\right) w_{m-s+1} E(s, n-1) .
$$

By adding the contributions given in equations (1) and (2) we obtain

$$
E(m, n)=\sum_{r}\left(\begin{array}{l}
n \\
r
\end{array}\right) w_{n-r+1} E(m-1, r)+n \sum_{s}^{m-2}\left(\begin{array}{c}
m-1 \\
s
\end{array}\right) w_{m-s+1} E(s, n-1) .
$$

It is not difficult to deduce a generating function for $E(m, n)$ from this recurrence relation. By writing $E\left(K_{m, n} ; \mathbf{w}, u, v\right)$ for the generating function of $E(m, n)$, we get

$$
E\left(K_{m, n} ; \mathbf{w}, u, v\right)=\exp \left[\sum_{\alpha}\left(w_{\alpha+1} \frac{u^{\alpha} v}{\alpha !}\right)+\sum_{\beta}\left(w_{\beta+1} \frac{u v^{\beta}}{\beta !}\right)\right] .
$$

By extracting the coefficient of $u^{m} v^{n}$ we can obtain $E(m, n)$. Hence we have the following result.

THEOREM 10. (1) The star polynomial of $K_{m, n}$ is

$$
E\left(K_{m, n} ; \mathbf{w}\right)=m ! n ! \sum\left[\prod_{\alpha, \beta} \frac{1}{j_{\alpha} ! j_{\beta} !}\left(\frac{w_{\alpha+1}}{\alpha !}\right)^{j_{\alpha}}\left(\frac{w_{\beta+1}}{\beta !}\right)^{j_{\beta}}\right],
$$

where the summation is taken over all solutions of

$$
\sum_{\alpha} \alpha j_{\alpha}=m \text { and } \sum_{\beta} \beta j_{\beta}=n .
$$

(2) Its generating function is

$$
E\left(K_{m, n} ; \mathbf{w}, u, v\right)=\exp \left[\sum_{\alpha}\left(w_{\alpha+1} \frac{u^{\alpha} v}{\alpha !}\right)+\sum_{\beta}\left(w_{\beta+1} \frac{u v^{\beta}}{\beta !}\right)\right], \quad \text { and }
$$

(3) $E\left(K_{m, n} ; \mathbf{w}\right)$ satisfies the recurrence relation

$$
E(m, n)=\sum_{r}\left(\begin{array}{l}
n \\
r
\end{array}\right) w_{n-r+1} E(m-1, r)+n \sum_{s}^{m-2}\left(\begin{array}{c}
m-1 \\
s
\end{array}\right) w_{m-s+1} E(s, n-1) .
$$

We can obtain the generating function and a recurrence relation for the simple star polynomial of $K_{m, n}$ directly from equation (4) by putting $\mathbf{w}=$ $(w, w, w, \ldots)$. This yields

COROllary 10.1 .

(1) $E\left(K_{m, n} ; w, u, v\right)=\exp [w v \exp (u)+w u \exp (v)-u v w]$ and

(2) $E(m, n)=w\left[\sum_{r}\left(\begin{array}{l}n \\ r\end{array}\right) E(m-1, r)+n \sum_{s}\left(\begin{array}{c}m-1 \\ s\end{array}\right) E(s, n-1)\right]$. 
An expression for $E\left(K_{m, n}: w\right)$ can be obtained from (1) of Theorem 10 by putting $\mathbf{w}=(w, w, w, \ldots)$. However a simpler formula is obtainable from Corollary 10.1 .

Let us write $E(m, n)$ for $E\left(K_{m, n} w\right)$. Then

$$
\begin{aligned}
\sum_{m, n} E(m, n) \frac{u^{m} v^{n}}{m ! n !}= & \exp [w v \exp (u)+w u \exp (u)-u v w] \\
= & \exp (w v \exp u) \cdot \exp (w u \exp v) \cdot \exp (-u v w) \\
= & \sum_{r} \frac{w^{r} v^{r}}{r !}\left(\sum_{i} \frac{u^{i} r^{i}}{i !}\right) \cdot \sum_{s} \frac{w^{s} u^{s}}{s !} \\
& \times\left(\sum_{j} \frac{v^{j} s^{j}}{j !}\right) \cdot \sum_{k}(-1)^{k} \frac{u^{k} v^{k} w^{k}}{k !} \\
= & \sum_{r} \sum_{i} \sum_{s} \sum_{j} \sum_{k}(-1)^{k} \frac{w^{r+s+k} r^{i} s^{j} u^{i+s+k} v^{j+r+k}}{r ! i ! s ! j ! k !}
\end{aligned}
$$

By equating coefficients of $u^{m} v^{n}$, we get

$$
\frac{E(m, n)}{m ! n !}=\sum_{r} \sum_{s} \sum_{k}(-1)^{k} \frac{w^{r+s+k} r^{m-s-k} s^{n-r-k}}{r !(m-s-k) ! s !(n-r-k) ! k !} .
$$

It follows by putting $r+s+k=p$, that

$$
E(m, n)=\sum_{p} w^{p} \sum_{\substack{r+s+k \\=p}}(-1)^{k} \frac{m ! n ! r^{m-s-k} s^{n-r-k}}{(m-s-k) !(n-r-k) ! r ! s ! k !}
$$

By multiplying top and bottom of the RHS by $(s+k) !(r+k)$ ! we obtain

THEOREM 11.

$$
E\left(K_{m, n} ; w\right)=\sum_{p} w^{p} \sum(-1)^{k} k !\left(\begin{array}{c}
m \\
s+k
\end{array}\right)\left(\begin{array}{c}
n \\
r+k
\end{array}\right)\left(\begin{array}{c}
s+k \\
k
\end{array}\right)\left(\begin{array}{c}
r+k \\
k
\end{array}\right) r^{m-s-k} s^{n-r-k},
$$

where the second summation is taken over all solutions of $r+s+k=p$.

The following is an immediate corollary of the above theorem.

COROLlaRY 11.1. The number of labelled $m$ by $n$ bipartite forests that consist of $p$ stars is

$$
\sum(-1)^{k} k !\left(\begin{array}{c}
m \\
s+k
\end{array}\right)\left(\begin{array}{c}
n \\
r+k
\end{array}\right)\left(\begin{array}{c}
s+k \\
k
\end{array}\right)\left(\begin{array}{c}
r+k \\
k
\end{array}\right) r^{m-s-k} s^{n-r-k}
$$

where the summation is taken over all non-negative integral solutions of $r+s+$ $k=p$.

9. Node disjoint decompositions of $\boldsymbol{K}_{\boldsymbol{m}, \boldsymbol{n}}$. The following result can be immediately deduced from Corollary 11.1 . 
CoRollary 11.2. $K_{m, n}$ can be decomposed into $p$ stars if $\exists$ non-negative integers $r, s$ and $k$ such that

(i) $p=r+s+k$

(ii) $s+k \leq m$

(iii) $r+k \leq n$.

The number of ways of decomposing $K_{m, n}$ into $p$ stars is given in Corollary 11.1. Notice that if we put $m=1$ in this corollary then we get that the number of ways of decomposing an $n$-star into $p$ stars is $\left(\begin{array}{c}n \\ p-1\end{array}\right)$ as is otherwise obvious.

The number of ways of decomposing $K_{m, n}$ into $r$ stars could be easily obtained by straightforward combinatorial techniques. It is also obvious that $K_{m, n}$ is $r$-star decomposable if and only if $m=r n$ or $n=m r$. For completeness, we add the following result

Corollary 11.3.

(i) $K_{m, n}$ is $r$-star decomposable if and only if $n=r m$ or $m=r n$.

(ii) If $n=r m$, the number of $r$-star decompositions of $K_{m, n}$ is

$$
m ! \prod_{i=1}^{m-1}\left(\begin{array}{c}
n-i r \\
r
\end{array}\right) .
$$

10. Conclusion. The star polynomial is a special type of $F$-polynomial (See Farrell [3]) in which the members of the family are stars. We have shown that the star polynomial could be helpful in obtaining results about node-disjoint decompositions of complete graphs and complete bipartite graphs into stars. Our results parallel those of Cain [2] and Ae et al. [1] in which edge-disjoint decompositions are considered. It might be possible to use the polynomials in order to obtain results about node-disjoint decompositions of other classes of graphs, for example wheels and ladders. Since the polynomials contain a wealth of information about the stars in the graph, they might be useful in any investigation of the star subgraphs of graphs.

\section{REFERENCES}

1. Tadashi Ae, Seigo Yamamoto, Noriyosha Yoshida, Line-disjoint Decomposition of Complete Graphs into stars, J. Comb. Theory Ser. B (to appear)

2. Pauline Cain, Decomposition of Complete Graphs into Stars, Bull. Austral. Math. Soc. Vol. 10 (1974), 23-30.

3. E. J. Farrell, On a General Class of Graph Polynomials J. Comb. Theory Ser. B (to appear).

4. F. Harary, Graph Theory, Addison-Wesley Pub. Co. Inc., Reading, Mass. (1969).

DePt. OF MATHEMATICS,

UNIVERSITY OF THE WEST INDIES,

St. Augustine,

TRINIDAD, W.I. 